\title{
VISÃO COMPUTACIONAL APLICACADA À METROLOGIA DIMENSIONAL AUTOMATIZADA: CONSIDERAÇÕES SOBRE SUA EXATIDÃO*
}

\author{
Flávio F. Feliciano ${ }^{1}$ \\ Igor L. de Souza ${ }^{2}$ \\ Fabiana R. Leta ${ }^{3}$
}

\begin{abstract}
Resumo: O presente artigo tem por objetivo tratar de algumas técnicas de Visão Computacional, que podem ser úteis em aplicações de automação. Estas aplicações incluem: medição de peças, inspeção visual, análise de conformidade, etc. Sistemas de Visão Computacional em medições sem contato são especialmente úteis quando se têm objetos pequenos ou de geometria complexa, nos quais instrumentos convencionais não podem ser utilizados. Nestes tipos de situações, em alguns casos é possível utilizar Máquinas de Medição por Coordenadas (MMC). Além do valor de MMC ser elevado, nem sempre é simples transportar a peça para máquina. Nestes casos sistemas de inspeção e medição por imagem são boas alternativas. O sistema proposto tem como principais objetivos minimizar erros inerentes aos operadores e facilitar processos que requerem instrumentos/equipamentos mais complexos de medição. As seguintes premissas são feitas: (1) os objetos devem ter pequena espessura; e, (2) blocos padrão são usados para conversão de unidades. Neste artigo, apresentam-se os algoritmos desenvolvidos para realizar as medições de objetos retangulares com furos. Os resultados obtidos são comparados com aqueles obtidos por instrumentos convencionais. São destacadas as dificuldades existentes em sistemas automatizados de medição por imagem, a exatidão destes e a importância de compreender tais problemas ao usá-los para fins industriais. $\mathrm{O}$ artigo não pretende esgotar o assunto, mas apenas apresentar uma reflexão sobre medições em imagens.
\end{abstract}

Palavras chave: Visão Computacional, Metrologia por imagem, Inspeção automatizada, Exatidão.

Abstract: This paper uses some Computer Vision techniques to measure parts and discusses ordinary difficulties of automated inspection. The parts conformity analysis using a non-contact measurement system has been used specially to small objects, where accurate instruments are not so simple to be used. In these cases Coordinate Measuring Machine (CMM) can be used. However, in many times it is impossible to move the object to the CMM. In this case an image-automated inspection should be a good alternative. The proposed system consists in a CCD camera positioned in the upper viewer of the object. Some considerations must be pointed out: (1) the object should be thin, to reduce the height influence in the measurement; (2) standard gages must be used to convert pixels units to millimeters units. We present the algorithms developed to make dimensional measurements in industrial parts. A rectangle object with three holes is analyzed. The obtained results are compared with conventional instruments measurements. We highlight the difficulties concerned to image automated inspection, discussing accuracy and the relationships among images and measurements.

Keywords: Computer vision, Metrology by image, Automated inspection, Accuracy.

\footnotetext{
${ }^{1}$ Universidade Federal Fluminense, Programa de Pós-Graduação em Engenharia Mecânica

${ }^{2}$ Universidade Federal Fluminense, Departamento de Engenharia Mecânica

3 Universidade Federal Fluminense, Programa de Pós-Graduação em Engenharia Mecânica, Laboratório de Metrologia Dimensional e Computacional, fabiana@lmdc.uff.br, Departamento de Engenharia Mecânica

*Este artigo foi baseado no trabalho publicado no 18th International Congress of Mechanical Engineering em 2005.

ENGEVISTA, v. 7, n. 2, p. 38-50, dezembro 2005 


\section{INTRODUÇÃO}

$\mathrm{O}$ uso de inspeções automatizadas na indústria vem se tornando cada vez mais uma solução interessante para análise de conformidade de peças. Em processos de manufatura devem ser avaliados: tolerâncias dimensionais, tolerâncias geométricas, ajustagem, incertezas e defeitos de fabricação. Com a evolução e redução dos preços das câmeras digitais, torna-se atrativo investir em sistemas de Visão Computacional para realização de medições e inspeções. Sistemas deste tipo podem oferecer exatidão e repetitividade em medições sem contato, especialmente por eliminar aspectos como subjetividade, fadiga, lentidão e custo associados à inspeção humana.

Em sistemas de medição por imagem automatizados, o problema principal consiste em saber combinar a tecnologia a uma aplicação específica de uma maneira econômica e com a qualidade requerida. Não há um sistema que resolva todos os desafios industriais, os sistemas são específicos e configurados para cada aplicação. O desenvolvimento de sistemas de visão industriais robustos e com a necessária exatidão torna-se, portanto, um desafio.

Sistemas de Visão Computacional envolvem técnicas de Processamento Digital de Imagens. Existem duas abordagens comuns nestes sistemas, a primeira refere-se a aplicações de reconhecimento e a segunda a inspeção automatizada (Fig. 1). Em um sistema para reconhecimento devem-se extrair características dos objetos da imagem e usar algum tipo de inteligência computacional para proceder a distinção entre os objetos. Neste caso não é fundamental obter valores exatos destas características, por outro lado em sistemas de inspeção a exatidão é fundamenta. A Metrologia por imagem consiste no processo automatizado de inspeção/medição, e engloba conceitos de Metrolgia e de Visão Computacional. É a medição sem contato que utiliza algoritmos computacionais.

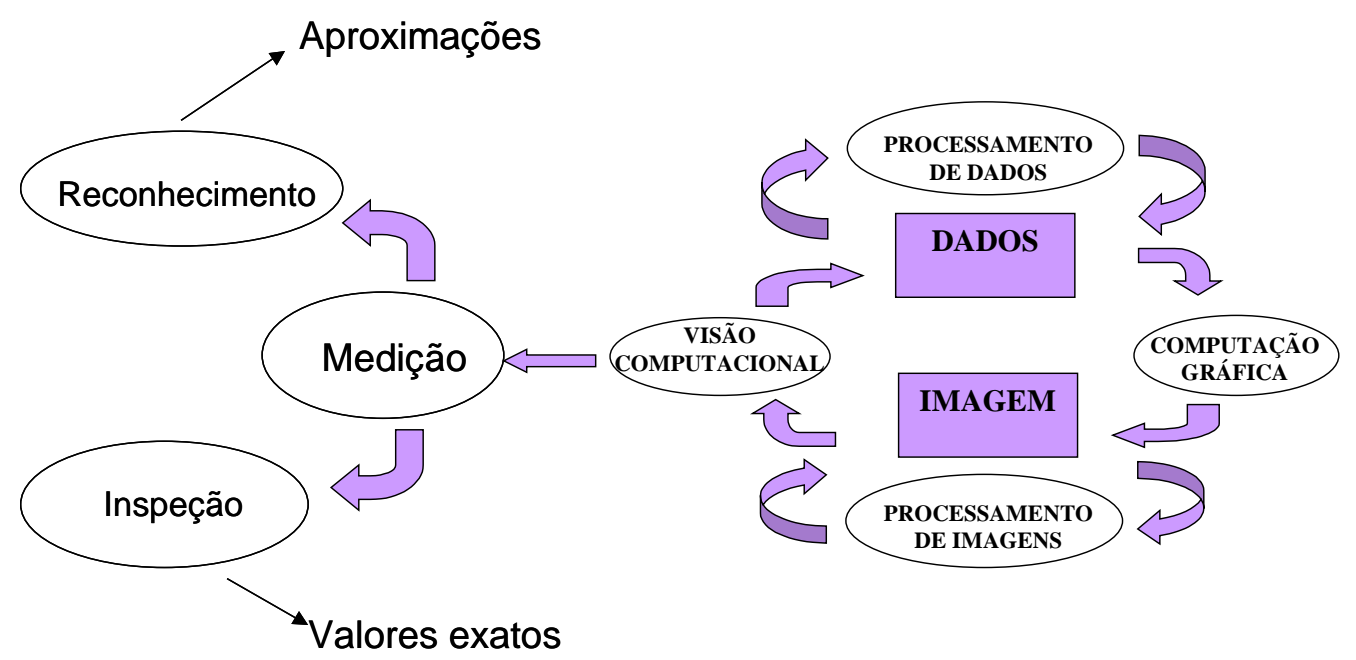

Figura 1- Sistema de Visão Computacional para medição (baseada em Gomes e Velho, 1994)

Neste contexto, podem-se destacar algumas aplicações industriais: identificação de erros de peças para controle de qualidade; orientação de robôs; rastreabilidade de materiais em linhas de produção; medição de peças. Medições por imagem possuem grande relevância em muitas aplicações. Luo et al. (2004) combinam Visão Computacional, interferometria laser e uso de máquina de medição por coordenadas para desenvolver um sistema de inspeção. Eles notaram que este tipo de sistema aumenta a exatidão das MMC. O trabalho de Khotanzad et al. (1994) descreve um sistema para inspeção automática de placas de circuito impresso. Christy et al. (2005) apresentam as técnicas de processamento de imagens utilizadas para 
avaliação da qualidade da superfície de pedaços de madeira. Liguori et al (2001) apresentam uma aplicação em imagens médicas, o objetivo é medir em imagens de utrasom a espessura de artérias. Pode-se observar que o campo de aplicação de sistemas de Visão Computacional é bem amplo, o que leva à necessidade de se estudar a qualidade de seus resultados. Muitos destes trabalhos não consideram a exatidão da medida através de imagens. De Santo et al. (2004) apresentam um estudo sobre a avalição de incerteza de medições por imagem.

Apesar do elevado nível de desenvolvimento tecnológico no campo da manufatura, ainda é difícil obter peças perfeitas. Para gerenciar tal limitação torna-se necessário utilizar tolerâncias dimensionais e geométicas que permitam a intercambialidade das peças. Em alguns casos a rejeição de peças se dá por uma escolha inadequada do sistema de medição e não por questões de não conformidade.

Neste contexto, este artigo tem por objetivo apresentar uma proposta de um sistema de Visão Computacional voltado para a medição de objetos, visando a análise de sua conformidade. Num processo automatizado uma inspeção visual deste tipo de peça pode consumir muito tempo e levar erros humanos inadmissíveis, que acarretam prejuízos na linha de produção.

Para o desenvolvimento do sistema são estudadas técnicas de extração das características dimensionais de objetos em imagem. Apresenta-se um estudo de caso envolvendo alguns problemas possíveis de existirem em um sistema automático de medição. Discutem-se os cuidados a serem tomados neste tipo de sistema e a incerteza envolvida.

\section{SISTEMA DE MEDIÇÃO POR IMAGEM}

\subsection{Aspectos gerais}

Uma imagem é descrita por uma matriz $\mathrm{NxM}$ de valores de pixel (picture element) inteiros positivos, que indica a intensidade de cor em cada posição $(i, j)$ da imagem. Um dado pixel $(p(i, j))$ na posição $(n, m)$ possui um valor numérico que representa o valor da iluminação na área correspondente na imagem.

Uma imagem usualmente contém alguns objetos. Uma aplicação de visão usualmente envolve computar certas propriedades de um objeto, e não a imagem como um todo. Para computar propriedades de um objeto, objetos individuais precisam primeiramente ser identificados como objetos separados; então as propriedades de objetos podem ser computadas.

Assim, um sistema de medição por imagem pode ser dividido nas seguintes etapas: (1) aquisição; (2) pré-processamento; segmentação de objetos; (4) reconhecimento dos objetos de interesse; e, (5) medição (Fig. 2). $\mathrm{Na}$ primeira etapa é necessário o uso de um bom sistema de iluminação e câmera com boa resolução. A aplicação das técnicas de processamento de imagens ocorre nas etapas 2 a 4, com o objetivo de preparar a imagem para a realização das medições.

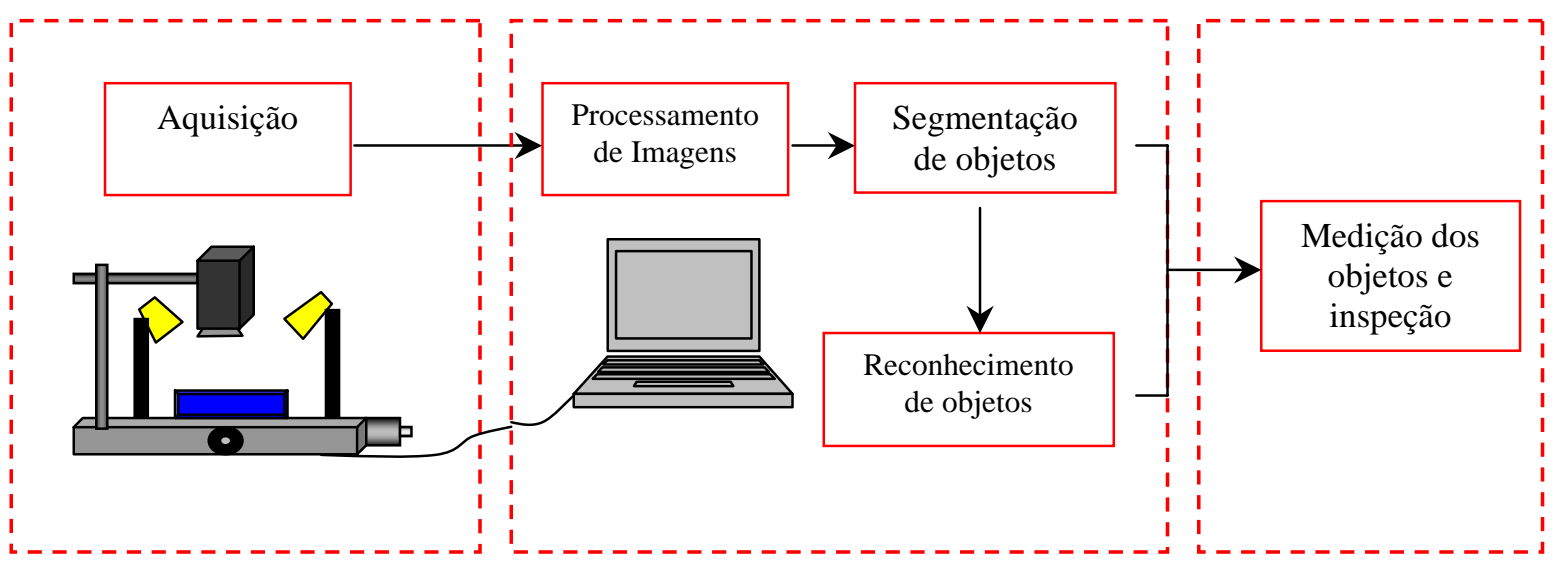

Figura 2 - Visão global do sistema. 
O pré-processamento deve ser realizado para aprimorar a qualidade da imagem, reduzindo ruidos inerentes ao processo de aquisição. $O$ processo de segmentação ou limiarização é utilizado para separar as áreas de interesse, que consistem nas peças a serem medidas. $\mathrm{O}$ processo mais simples de segmentação é chamado de não-contextual, pois não leva em consideração as relações entre os pixels e regiões (Schalkoff, 1989). Neste caso separam-se os objetos do fundo, mudando a cor destes para preta e o do fundo para branca, tem-se então uma imagem binária, que é muito usual nas diversas aplicações de Visão Computacional.

\subsection{Pré-processamento}

Para interpretar uma imagem, as variações nos valores de intensidade devem ser analisadas. Os números de níveis de quantificação usados mais comumente para representar intensidades de imagens são de 256 diferentes níveis de cinza, o que implica um maior esforço computacional e de armazenamento. Estas limitações encorajaram o uso de sistemas de visão binária (uma imagem binária contém apenas dois níveis de cinza).

Necessidades menores de memória e tempos de execução mais rápidos não são as únicas razões para estudar sistemas de visão binária. Muitas técnicas desenvolvidas para estes sistemas também são aplicáveis para sistemas de visão que usam imagens de escala de cinza. Em geral, sistemas de visão binária são úteis em casos onde o contorno contém informação suficiente para permitir reconhecimento de um objeto e onde o ambiente pode ser controlado adequadamente. Para obter um bom contorno, os objetos devem ser separados facilmente do fundo. O sistema de visão binária geralmente usa um limiar para separar objetos do fundo. O valor apropriado deste limiar depende da iluminação e das características reflexivas dos objetos. Para a limiarização ser efetiva na separação objetofundo, é necessário que o objeto e o fundo tenham contraste suficiente e que se saiba os níveis de intensidade tanto dos objetos quanto do fundo. Em um esquema de limiarização fixa, estas características de intensidade determinam o valor do limiar (Ramesh et al, 1995).

A limiarização distingue os objetos do fundo em uma imagem. Em alguns casos tornase necessário distinguir mais de um objeto ou mais de uma característica no objeto. Neste caso, pode-se optar por operações de detecção de vizinhos similares. Em uma imagem digital representada numa grade quadricular, um pixel tem uma fronteira comum com quatro pixels e compartilha um canto comum com quatro pixels adicionais. Diz-se que dois pixels são 4vizinhos se eles compartilham uma fronteira comum (Marques Filho e Vieira Neto, 1999). Semelhantemente, dois pixels são 8-vizinhos se eles compartilham pelo menos um canto. Por exemplo, um pixel no local $[\mathrm{i}, \mathrm{j}]$ tem 4-vizinhos [i+1,j], [i-1,j], [i,j+1] e [i,j-1]. Os 8-vizinhos do pixel incluem os 4-vizinhos mais $[\mathrm{i}+1, \mathrm{j}+1]$, $[i+1, j-1],[i-1, j+1]$ e $[i-1, j-1]$.
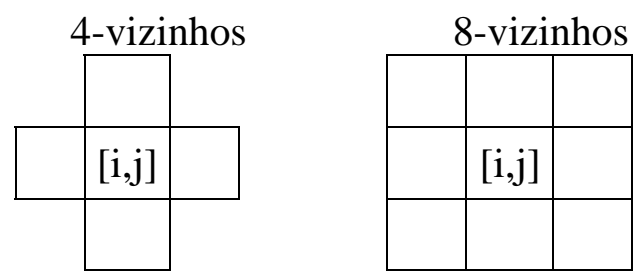

Figura 3. Sistemas de vizinhança de um ponto.

É possível distinguir mais de um objeto na mesma imagem apenas considerando o sistema de vizinhança dos pontos, sabe-se que cada objeto tem todos os pixels referentes a ele ligados uns aos outros, então ao considerar uma cor de fundo basta observar que se um aglomerado de pixels não está conectado a outro por nenhum pixel de mesmo tom estes representam objetos diferentes.

Alguns cuidados devem ser tomados ao utilizar-se esta metodologia. Na Fig.4 nota-se que utilizando o sistema 4-vizinhos tem-se um ponto que liga o quadrado ao circulo, o que acarreta que o círculo e o quadrado sejam considerados um único objeto. Neste caso, embora o custo computacional seja maior, é mais adequado o uso do método 8-vizinhos. 


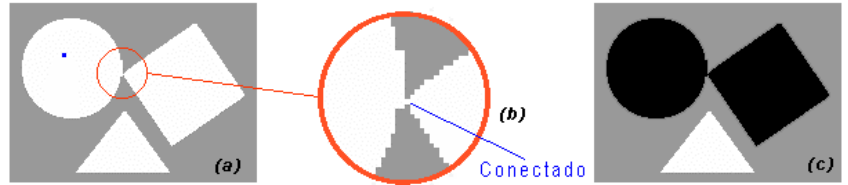

Figura 4 - (a) imagem original, (b) ampliação da área circulada em vermelho, (c) resultado do procedimento.

Estas metodologias serão úteis no estudo de caso apresentado, no qual tem-se uma peça retangular que apresenta três furos com diâmetros diferentes. Neste caso considerou-se cada furo da peça como objetos distintos e o programa tem de identificar que cada furo não está conectado ao outro ou ao fundo da imagem.

\subsection{Obtenção de características de objetos}

$\mathrm{Na}$ maioria das aplicações industriais, a localização da câmera e o ambiente são conhecidos, e o número de objetos diferentes não é grande. Depois de feita a separação de dois ou mais objetos dentro de uma imagem, pode-se programar o reconhecimento e extração de medidas de tais objetos.

Uma vez localizado o objeto, são desenvolvidos algoritmos que extraiam características do objeto que se encontra na imagem já limiarizada, ou seja, todos os processos descritos a seguir levam em consideração que a imagem original tem apenas dois tons (preto e branco).

Para identificar o contorno de um objeto, fundamental para análise da geometria, foram desenvolvidos dois métodos, um baseia-se em identificar quais pixels da imagem fazem fronteira com o fundo, o outro consiste em identificar um pixel que esteja no limite entre objeto e fundo e a partir dele faz-se uma varredura no sentido horário deste limite.

O primeiro é uma solução rápida que exige pouco processamento, porém não permite uma identificação seqüencial, simplesmente devolvendo uma imagem em que aparece somente o contorno. O segundo faz uma varredura ponto a ponto e por sua vez utiliza mais tempo de processamento que o primeiro, porém, nele é possível gerar uma tabela com as coordenadas de todos os pontos do contorno, o que pode ser útil em procedimentos posteriores (Feliciano et al, 2004).

\section{Características de circunferências}

Este método foi desenvolvido para que a partir de uma imagem de uma circunferência sejam extraídas as coordenadas do centro e o módulo do raio. Para definir o centro é usado o método de determinar centróides (Feliciano et al, 2004), que devolve as coordenada do centro da circunferência. Então, é feita uma varredura dos pontos do contorno e então é calculada a distância de cada ponto do contorno da circunferência ao centróide e o raio será dado pela média de todas estas distâncias.

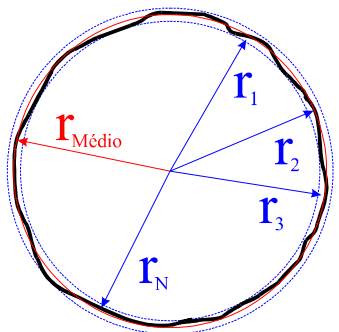

Figura 5. Exemplo de imperfeição da circunferência o raio médio é definido pela média de todos os raios calculados.

O interessante desta abordagem é que tornase possível comparar facilmente as medidas obtidas com a tolerância de circularidade definida.

\section{Identificação dos Vértices}

Os métodos que estão aqui descritos identificam vértices em objetos que contenham apenas arestas em linha reta e quando é conhecido o número de vértices cujas coordenadas deseja-se obter. Foram estudados dois métodos. $\mathrm{O}$ primeiro baseia-se em identificar os pontos do contorno com maior distância ao centróide da figura e o segundo faz uma varredura no contorno e identifica mudanças bruscas de direção (Feliciano e Souza, 2004).

Por limitação de espaço apresenta-se neste artigo apenas o segundo método que se mostrou mais eficiente. Este método tem por base identificar as retas que compõem as arestas da figura e com a intercessão das equações de retas destas temos um ponto em comum que é 
considerado um vértice. Conhecendo todos os pontos do contorno divide-se esta quantidade de pontos em intervalos iguais e é calculada a equação da reta e armazenado um ponto de cada pequeno intervalo, gerando uma tabela com os pontos e os coeficientes angulares de cada intervalo (Leta et al., 2005).

Tendo definido um ângulo de tolerância avalia-se a diferença entre cada coeficiente angular e o seu anterior até que se ache a diferença maior que a tolerância, assim é tirada a média dos coeficientes angulares e das distancias $\mathrm{x}$ e $\mathrm{y}$ dos pontos, gerando assim informações que definem uma reta. A busca prossegue até que se encontre uma nova diferença maior que a tolerância e assim por diante.

Cada reta definida corresponde a uma aresta e então os vértices são definidos na interseção destas arestas.

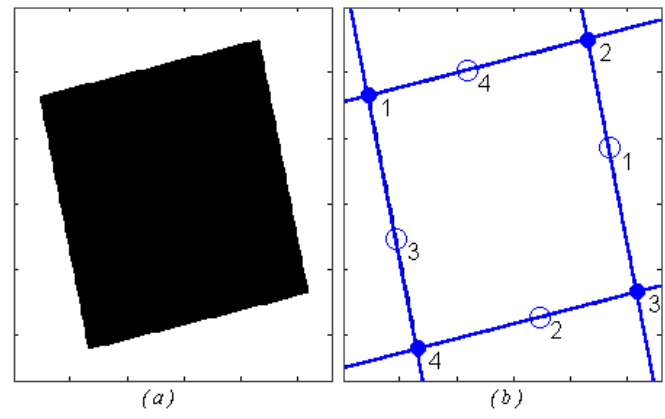

Figura 6. (a) figura original. (b) retas identificadas.

A partir da obtenção dos vértices do objeto, torna-se possível extrair comprimentos e avaliar algumas tolerâncias geométricas.

\subsection{Obtenção de características dimensionais e geométricas de objetos}

O estudo de caso apresentado trabalha com uma foto em tons de cinza de uma peça retangular que apresenta três furos com diâmetros diferentes Fig. 7. O objetivo do sistema é extrair automaticamente (sem a intervenção humana) as dimensões da borda da peça, o diâmetro de cada furo, e a posição do centro de cada furo em relação ao canto superior esquerdo da imagem, e conseqüentemente a distância do centro de cada furo as arestas laterais da figura. Estas informações são utilizadas para avaliação dimensional da peça, considerando ainda as tolerâncias dimensionais e geométricas da mesma. Neste caso considera-se cada furo da peça como um objeto distinto, utilizando-se a metodologia de vizinhança descrita previamente. Este resultado pode ser observado na Fig. 8.

A partir daí os vértices da chapa metálica e as circunferências dos furos podem ser identificados na figura (Fig. 9).

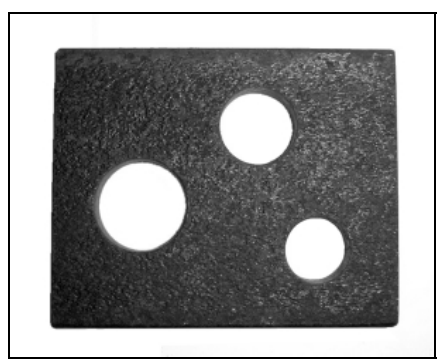

Figura 7. Foto da Peça em tons de cinza.

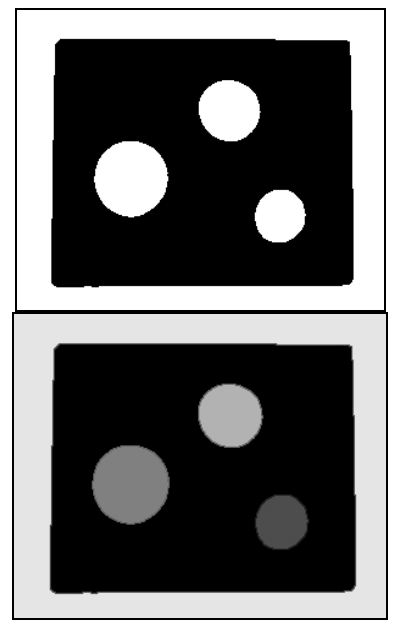

Figura 8 Imagem Limiarizada (preto e branco) e separação dos objetos por cor.

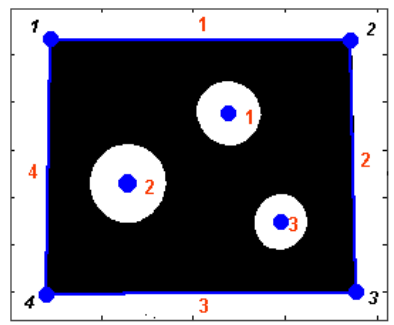

Figura 9. Resultado Final, vértices e centro dos furos apresentam-se marcados. 


\section{RESULTADOS}

Os resultados foram obtidos em unidades de pixels, para conhecer estes valores devemos primeiramente conhecer o fator de conversão pixels $x$ milímetros. Os erros destas medidas podem ser vistos em Leta et al. (2005).

Para obter o fator de conversão, um bloco padrão foi fotografado usando a câmera em diferentes alturas (Tab. 1). Pode-se notar que o fator de conversão $(\lambda)$ aumenta conforme a altura da câmera diminui. Considerando que $\lambda$ expressa a quantidade de pixels que corresponde a 1mm, a melhor posição de camera é aquela mais próxima ao objeto.

Para analisar a performance do sistema, uma peça foi estudada, usando a menor altura possível de câmera. A peça $C$ foi fotografada em diferentes posições (Fig. 10). Cabe ressaltar que a peça em estudo foi fabricada sem grande preocupação com a exatidão. Por esta razão atribuiram-se valores altos para tolerâncias de aceitação de conformidade. Mesmo que avaliadas por instrumentos convencionais, a peça seria descartada por falta de conformidade com o projeto. Em sistemas por imagem é importante avaliar os objetos em posições rotacionadas, pois o efeito de aliasing pode levar a erros inerentes ao processo de digitalização.

Tabela 1 Altura da câmera, comprimento do bloco padrão em pixels e fator de conversão (Rbloco padrão $-50 \mathrm{~mm}$ ).

\begin{tabular}{|c|c|c|c|}
\hline Imagem & $\begin{array}{c}\text { Altura } \\
\text { (mm) }\end{array}$ & $\begin{array}{c}\text { Bloco } \\
\text { padrão } \\
\mathbf{P} \text { (pixel) }\end{array}$ & $\begin{array}{c}\text { Fator de } \\
\text { conversão } \\
\lambda=\frac{P}{R} \\
\text { (pixel/mm) }\end{array}$ \\
\hline $\mathrm{a}$ & 100 & 496 & 9,92 \\
\hline $\mathrm{b}$ & 150 & 363 & 7,26 \\
\hline $\mathrm{c}$ & 200 & 275 & 5,50 \\
\hline $\mathrm{d}$ & 250 & 228 & 4,56 \\
\hline $\mathrm{e}$ & 300 & 197 & 3,94 \\
\hline
\end{tabular}

Tabela 2. Especificação.

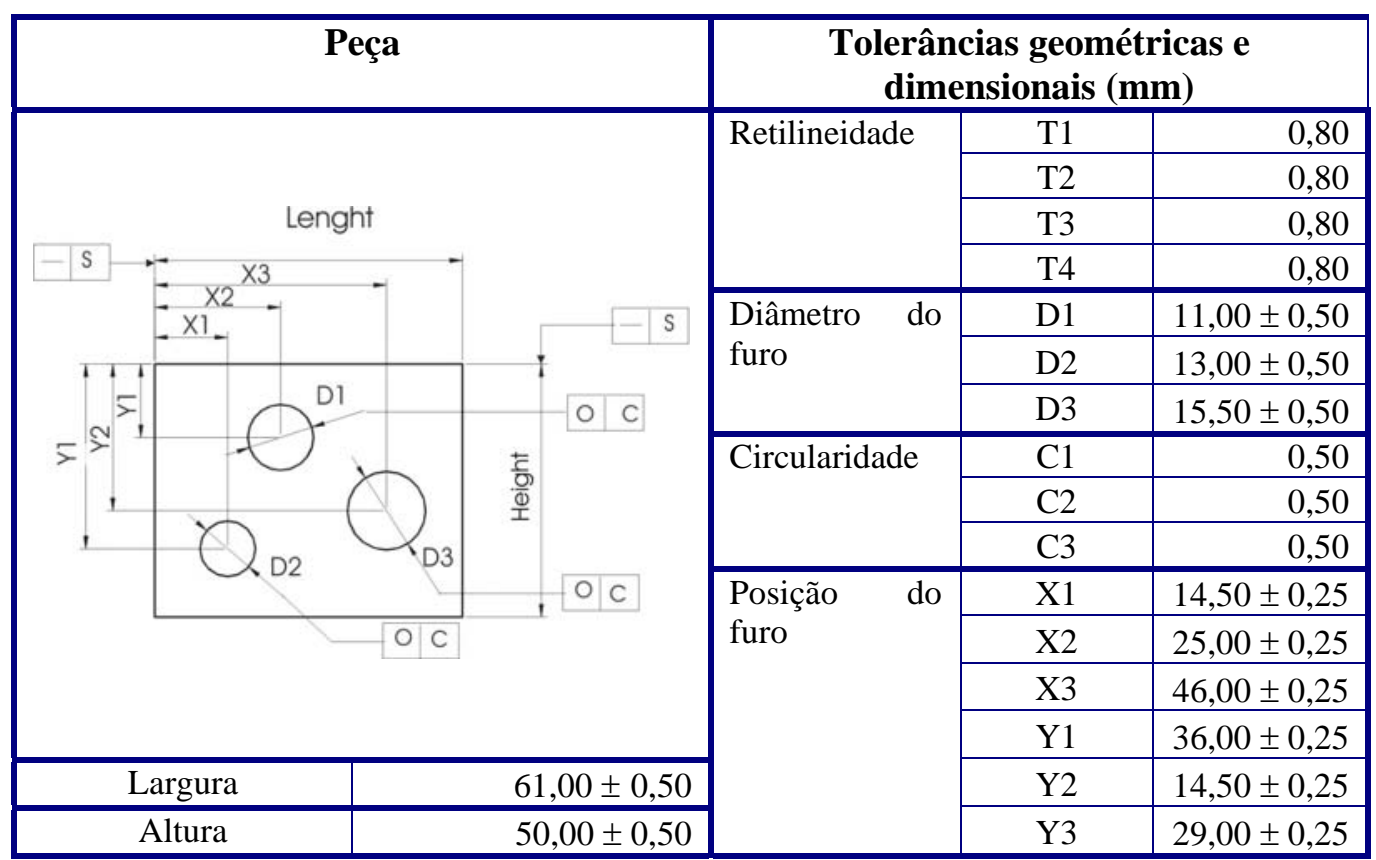


Tabela 3. Medidas obtidas.

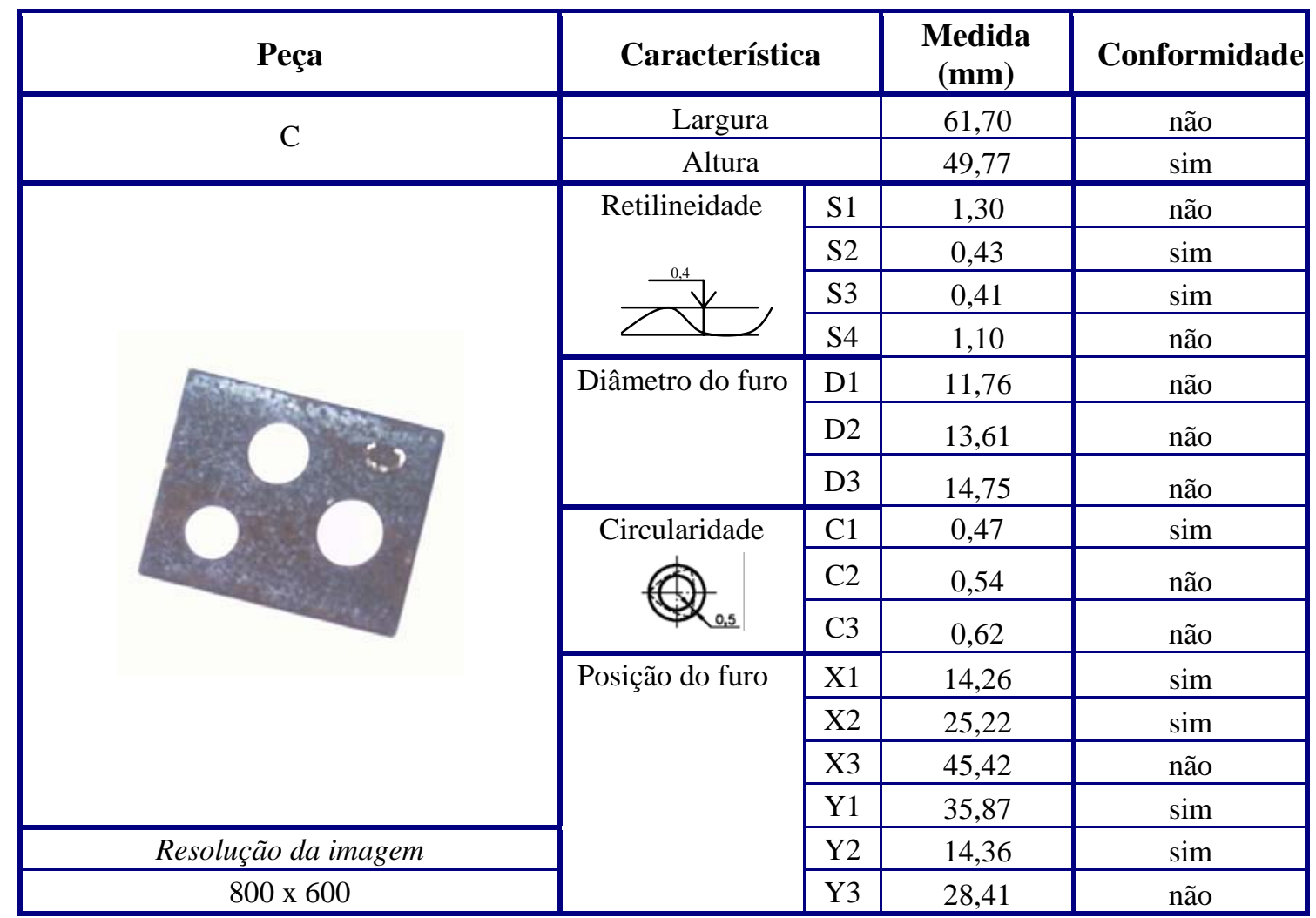

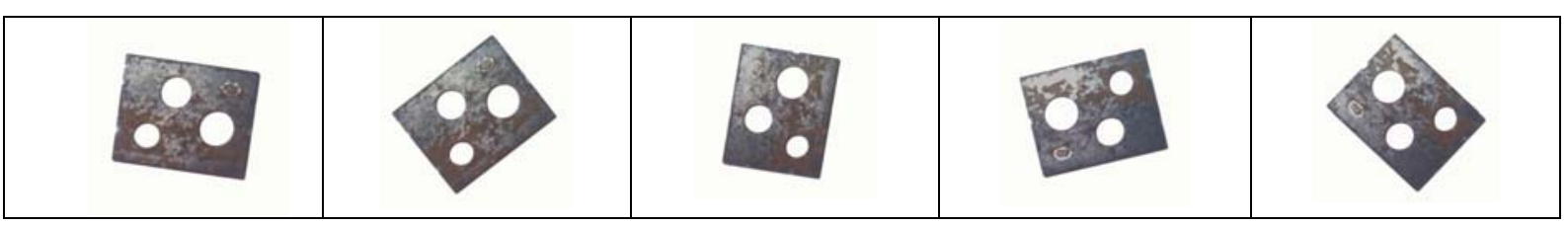

Figura 10. Digitalização da peça em posições diferentes.

\section{ERROS EM PROCESSOS MEDIÇÃO POR IMAGEM}

Todas as técnicas aqui apresentadas são utilizadas para trabalharem com objetos contidos em imagens digitais. Portanto, as características dimensionais obtidas dos mesmos são extraídas, em um primeiro momento, em unidade de pixels. Para uma aplicação real, torna-se necessário o uso de cálculos para convertê-las em unidades reais de medida do SI. Para isso, deve-se conhecer a resolução das fotos tiradas. Quanto maior a resolução, mais exatas serão as medidas, já que o números de pixels dentro da mesma área é aumentado. É preciso também saber a distância da câmera ao objeto, ter o devido controle da iluminação do ambiente para evitar problemas de sombra (Fig. 11). Outros fatores que devem ser considerados para que estas técnicas automatizadas (sem a intervenção do usuário) possam ser utilizadas com sucesso podem ser vistos em Leta et al. (2005) e Feliciano e Souza (2004), como por exemplo: imperfeições de superfícies e objetos em que a espessura afeta a medida (Fig. 11).

Sanando-se tais problemas, deve-se destacar que a solução por um sistema automatizado ainda é mais adequada do que sistemas por imagem em que o usuário define os pontos sobre o objeto a serem considerados para a medição. Nestes sistemas o usuário é levado a escolher os pontos extremos para medições, e, é a partir deste tipo de escolha que são gerados erros, pois são dependentes da percepção e treinamento do observador. 


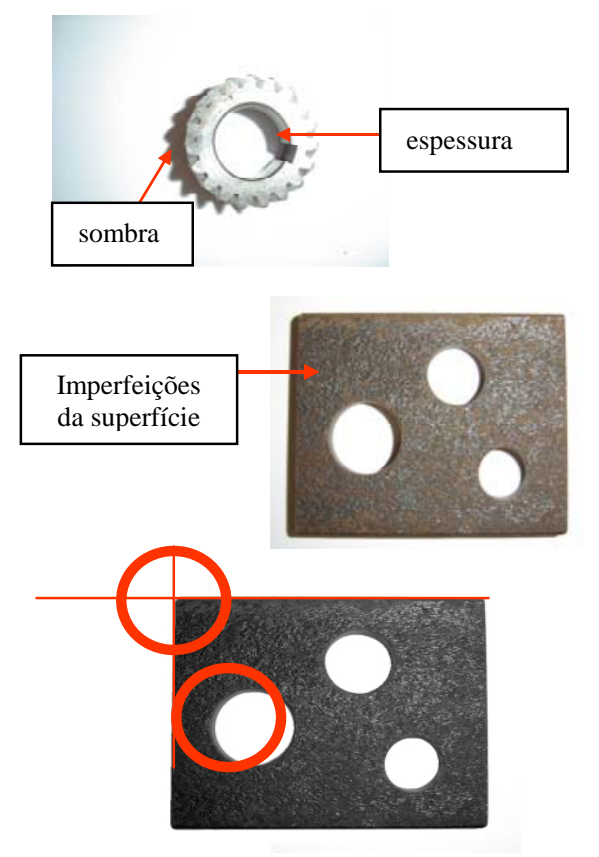

Figura 11. Problemas que podem gerar erros em medidas automáticas por imagens.

Observando-se a Fig. 12, há possibilidade do usuário escolher como início da medida do comprimento da peça pontos diferentes, já que a borda da peça não está claramente definida. O mesmo ocorre com relação à marcação do diâmetro, em especial devido à espessura da peça. O significado de marcar erradamente a borda para realizar uma medição pode ser mensurado quando se converte o número de pixels para a unidade de comprimento. Um erro de 1 pixel pode representar o equivalente a um erro de $0,25 \mathrm{~mm}$. Sendo que em geral não se erra em apenas um pixel. Ou seja, torna-se extremamente importante reduzir o erro do operador a partir de metodologias automáticas de medição.

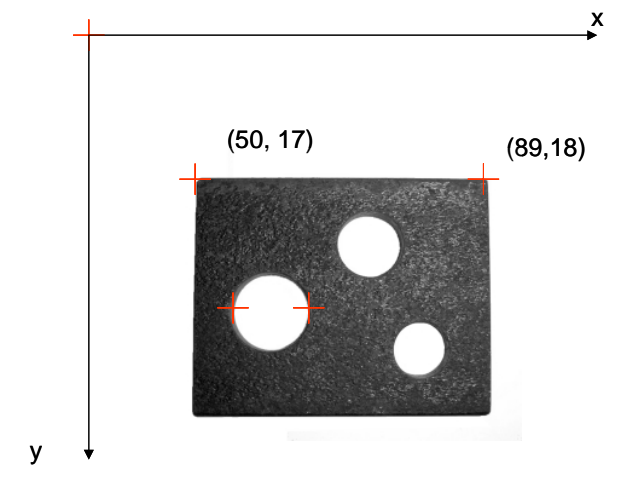

Figura 12. Seleção de pontos de marcação duvidosos.

\section{ANÁLISE DE INCERTEZAS EM MEDIÇÕES POR \\ VISÃO \\ COMPUTACIONAL}

Em aplicações de medição, principalmente na realização de calibrações, é necessário um estudo criterioso para a expressão da incerteza de medição. Nesta expressão são tratadas informações e critérios para avaliação dos componentes que influenciam o resultado de uma medição. A incerteza expressa, resumidamente, o valor provável do erro de uma medição.

Muitas vezes os resultados de uma medição devem ser comparados com uma tolerância ou limites definidos por alguma especificação ou regulamentação. Nestes casos, o conhecimento da incerteza da medida permite decidir se o resultado obtido está dentro dos limites preestabelecidos. O cálculo da incerteza depende do conhecimento detalhado da natureza do mensurando, do padrão, das influências externas e do processo de medição.

Para este estudo de medição através de Visão Computacional torna-se importante, também, obter a incerteza deste processo de medição, que está inserido no campo da Metrologia Computacional. Para isto foram estudados os possíveis fatores que podem contribuir, de alguma forma, para os erros de medição. Além disso, é necessário, primeiramente, destacar a diferença existente entre a incerteza do tipo A e a incerteza do tipo B.

\section{Incerteza Tipo A}

Em Metrologia, pode-se afirmar que a melhor estimativa de uma grandeza, que varia aleatoriamente, é a média aritmética das $n$ medidas efetuadas e calculada por:

$$
\bar{x}=\frac{\sum x_{i}}{n}
$$

Os valores $\mathrm{x}_{\mathrm{i}}$ diferem entre si por causa de efeitos aleatórios que ocorrem durante as medições.

A variância estimada $s^{2}\left(x_{i}\right)$ ou o desvio padrão estimado $\mathrm{s}\left(\mathrm{x}_{\mathrm{i}}\right)$ caracterizam a variabilidade dos valores medidos $\mathrm{x}_{\mathrm{i}}$, isto é, a dispersão dos mesmos em torno do valor médio. A expressão da variância é dada por: 


$$
s^{2}\left(x_{i}\right)=\frac{\sum\left(x_{i}-\bar{x}\right)^{2}}{n-1}
$$

A melhor estimativa da variância da média $\sigma^{2}(\bar{x})$, da grandeza a medir, é a variância experimental da média cuja expressão é:

$$
s^{2}(\bar{x})=\frac{s^{2}\left(x_{i}\right)}{n}
$$

O desvio padrão experimental da média serve para qualificar quanto o valor médio $\bar{x}$ representa a grandeza a ser medida X. Esta estimativa é tanto melhor quanto maior for o número de repetições efetuadas na medição.

A expressão da incerteza Tipo A, determinada a partir de $\mathrm{n}$ medições de uma grandeza $X$, para $v$ graus de liberdade e nível de confiança $\mathrm{p}=95 \%$, é dada por:

$$
i_{A}=I(\bar{x})= \pm t_{95 \%}(v) \times s(\bar{x})
$$

Onde:

- tp(v) é conhecido como t de student para um nível de confiança p

- v é o número de graus de liberdade, definido como n-1

\section{Incerteza Tipo B}

Incertezas deste tipo são determinadas a partir de informações acessórias e externas ao processo de medição. Estas informações podem ser obtidas de resultados de medições similares anteriores, experiência ou conhecimento do comportamento do instrumento, dados do fabricante, dados fornecidos por certificados de calibração, referências de manuais de instrução, etc.

São exemplos deste tipo de incerteza: gradiente de temperatura durante a medição; afastamento da temperatura ambiente em relação à temperatura de referência de $20 \mathrm{C}$, tipo do indicador: analógico ou digital, instabilidade na rede elétrica, paralaxe, incerteza do padrão, instabilidade do padrão, erros geométricos, deformações mecânicas, histerese, estabilidade temporal, etc.

Como mencionado anteriormente, uma análise de incertezas para este método de Visão Computacional torna-se necessária, uma vez que cada método de medição possui incertezas e fatores externos diferentes que podem contribuir para o erro. Além disto, pode-se observar a eficiência deste método e comparálos a outros métodos convencionais.

Para a avaliação da incerteza Tipo A, é preciso que haja um determinado número de medições feitas nas mesmas condições externas, uma vez que esta avaliação está relacionada com a repetitividade do processo. Uma alternativa para determiná-la foi fotografar uma mesma peça em orientações diferentes, ou seja, fazendo-a girar no plano em que se encontra, mantendo fixas a altura da câmera em relação ao objeto e a resolução da mesma. Deve-se lembrar que em aplicações reais as peças podem estar em orientações diferentes e aleatórias. Da mesma forma que em um processo de medição com um micrômetro, por exemplo, há uma grande probabilidade de se obter leituras diferentes de uma medida ao se repetir o processo algumas vezes.

Com isto, extrai-se a medição desejada em cada foto e calcula-se a incerteza utilizando-se a expressão da incerteza Tipo A (1). É preciso lembrar que quanto maior o número de fotosmelhor será a estimativa da incerteza do processo.

Para a estimativa da incerteza Tipo B, devese levar em conta fatores externos que podem contribuir para o erro. Foram destacados dois fatores que podem ser relevantes.

Primeiramente, ao se calibrar uma câmera para um processo, ou seja, fixar uma altura de câmera e extrair o fator de conversão por comparação com a medida real de uma peça de referência, há uma incerteza associada a este processo. Se a medida real conhecida da peça foi obtida por um outro processo de medição, a incerteza associada a este procedimento irá contribuir para a incerteza da medição computacional. Uma outra possibilidade é calibrar a câmera com uma foto de um bloco padrão. Neste caso a incerteza associada referese à incerteza do mesmo. O valor desta incerteza pode ser obtido no certificado de calibração que geralmente acompanha o conjunto de blocos padrão, quando o mesmo é adquirido. 
Uma segunda variável que cabe ser observada é a temperatura do ambiente de medição. Pode ser importante avaliar a incerteza associada ao afastamento da temperatura ambiente da temperatura de referência. Esta incerteza depende da grandeza da dimensão do mensurando e do afastamento da temperatura de medição da temperatura de referência $\left(20^{\circ} \mathrm{C}\right)$. Admitindo $\alpha$ como sendo o coeficiente de dilatação térmica do mensurando e $\Delta \mathrm{t}$ o afastamento da temperatura em relação a $20^{\circ} \mathrm{C}$, tem-se que o limite a será: $\mathrm{a}=\alpha \times \Delta \mathrm{t} \times \mathrm{L}$, onde L é o comprimento avaliado. O desvio padrão para $\mathrm{p}=68 \%$ será de:

$$
s\left(x_{i}\right)=\frac{a}{\sqrt{3}}
$$

Geralmente, a tolerância aceitável é de 2 C para cima e para baixo. Já em laboratórios de calibração ela é menor (cerca de 0,5 C). A relevância desta incerteza dependerá da aplicação e do ambiente. Se for um ambiente com uma temperatura alta (em uma linha de produção, por exemplo), este fator pode ser importante. Para um laboratório de calibração, este fator também deverá ser considerado.

A Tabela.4 mostra um resumo das variáveis e seus respectivos tipos de incerteza.

Uma análise criteriosa destas variáveis é fundamental para ser determinar o que pode ser feito para diminuir ao máximo a incerteza de medição por este método. A eficiência de um método de medição é tanto maior quanto menor for esta incerteza. Deve-se, em uma aplicação real, determinar quais variáveis podem ser controladas ou modificadas para a melhoria do processo. De posse destes valores, utilizam-se as equações (1), (2) e (3) para obter, respectivamente, os valores da variância, variância da média e da incerteza $\mathrm{U}_{\mathrm{r}}$, devido à repetibilidade, para cada medida. Os valores obtidos estão contidos na Tab. 5.

Tabela.4 - Variáveis e respectivos tipos de incerteza.

\begin{tabular}{|c|c|}
\hline Variável & Incerteza \\
\hline Medida da peça de referência & Tipo B \\
\hline $\begin{array}{c}\text { Procedimento de medição } \\
\text { computacional }\end{array}$ & Tipo A \\
\hline Temperatura ambiente & Tipo B \\
\hline
\end{tabular}

Porém, para se obter o valor total da incerteza do processo de medição $\mathrm{U}$, deve-se adicionar a contribuição da incerteza do bloco padrão $\mathrm{U}_{\mathrm{b}}$, que é de $0,53 \mu \mathrm{m}$, conforme mencionado anteriormente. Isto é feito através da seguinte relação:

$$
\mathrm{U} 2=\mathrm{Ur} 2+\mathrm{Ub} 2
$$

Onde: $\mathrm{U}=$ incerteza de medição

$$
\mathrm{U}_{\mathrm{r}}=\text { incerteza associada a repetitividade }
$$

$\mathrm{U}_{\mathrm{b}}=$ incerteza associada ao bloco padrão

A Tabela mostra os resultados obtidos, para cada medida da peça. Os valores se encontram com doze casas decimais, para que

\begin{tabular}{|c|c|c|c|c|}
\hline \multicolumn{2}{|c|}{ Medida } & Variância & Variância da média & Incerteza $U_{r}$ \\
\hline \multicolumn{2}{|c|}{ Comprimento } & 0,07044670 & 0,01408934 & 0,03916837 \\
\hline \multicolumn{2}{|c|}{ Largura } & 0,08572030 & 0,01714406 & 0,04766049 \\
\hline \multirow{3}{*}{ Diâmetro } & Furo 1 & 0,04278650 & 0,00855730 & 0,02378929 \\
\hline & Furo 2 & 0,08264350 & 0,01652870 & 0,04594979 \\
\hline & Furo 3 & 0,05520750 & 0,01104150 & 0,03069537 \\
\hline
\end{tabular}
se possa notar o efeito do cálculo.

Tabela 5 - Variância, Variância da média e Incerteza $U_{\mathrm{r}}$. 
Tabela 6 - Valores das incertezas.

\begin{tabular}{|c|c|c|c|c|}
\hline \multicolumn{2}{|c|}{ Medida } & Ur & Ub & $\mathbf{U}$ \\
\hline \multicolumn{2}{|c|}{ Comprimento } & 0,039168365200 & 0,00000053 & 0,039168365204 \\
\hline \multicolumn{2}{|c|}{ Largura } & 0,047660486800 & 0,00000053 & 0,047660486803 \\
\hline \multirow{3}{*}{ Diâmetro } & Furo 1 & 0,023789294000 & 0,00000053 & 0,023789294006 \\
\hline & Furo 2 & 0,045949786000 & 0,00000053 & 0,045949786003 \\
\hline & Furo 3 & 0,030695370000 & 0,00000053 & 0,030695370005 \\
\hline
\end{tabular}

É importante destacar que a diferença encontrada entre os valores de incerteza para cada medida (comprimento, largura e diâmetros), devido a diferentes variabilidades nas medições, se deve pelas irregularidades da peça. Portanto, ao se expressar a incerteza de medição para uma das medidas, deve-se associar o valor correspondente a cada uma.

Massimo et al (2000) descrevem as possíveis causas para a variabilidade das medidas na repetitividade, ou seja, onde as condições são mantidas fixas. Eles mencionam a incerteza proveniente da digitalização de uma imagem. Para a estimativa da expressão, os autores definiram a incerteza de um pixel de uma imagem UI (i, j) como o parâmetro que quantifica as mudanças aleatórias da intensidade I ( $\mathrm{i}, \mathrm{j}$ ) do próprio pixel. A incerteza pode ser medida como o desvio padrão $\sigma \mathrm{I}(\mathrm{i}, \mathrm{j})$ de uma série de $\mathrm{N}$ valores de I (i,j), obtidas por $\mathrm{N}$ aquisições da imagem em condições estacionárias de todos os parâmetros controláveis de influência.

Os autores destacam três causas prováveis para tal:

a) a quantização da imagem, do mundo contínuo real para o mundo discreto computacional, relacionada à quantização espacial e de intensidade;

b) a presença de vibrações, que contribuem para a quantização de intensidade;

c) a variabilidade intrínseca da origem da luz (instabilidade, luz tremeluzindo, etc.);

Os autores modelam expressões para a estimativa de tais incertezas, porém estas modelagens não foram consideradas no presente artigo.

\section{CONSIDERAÇÕES FINAIS}

A escolha por técnicas medição automáticas de por imagem requer uma avaliação detalhada sobre os diferentes algoritmos possíveis de serem implementados, bem como as condições de iluminação e do objeto a ser medido. Podese notar que para algumas aplicações, pode haver mais de uma técnica diferente. Cabe ao usuário decidir quais técnicas se enquadram de modo mais satisfatório para sua aplicação.

Este trabalho teve o objetivo de apresentar, sem esgotar o assunto, algumas técnicas de processamento e análise de imagens focadas em Metrologia por imagem. Observa-se que os algoritmos de Visão Computacional têm a capacidade de facilitar bastante o trabalho humano na indústria, além de possibilitar a realização de tarefas de modo mais preciso e eficaz, aumentando consideravelmente a produtividade e a garantia da qualidade dos produtos fabricados. Porém, se utilizada sem a avaliação e os cuidados adequados pode resultar em medidas inexatas.

\section{AGRADECIMENTOS}

Os autores agradecem à FAPERJ pelo apoio ao projeto temático intitulado Metrologia Baseada em Visão Computacional, à CAPES e ao MEC-SESU pelas bolsas de mestrado e PET de um dos autores. Agradecem também ao técnico Alain Rangel pelo apoio na realização do estudo de caso.

\section{REFERÊNCIAS}

Christy, A.G., Senden, T.J. e Evans, P.D. Automated measurement of checks at wood surfaces. Measurement, Vol. 37, pp. 109-118, 2005. 
De Santo, M., Liguori, C., Paolillo, A. e Pietrosanto, A. Standard uncertainty evaluation in image-based measurements. Measurement, Vol. 36, pp. 347-358, 2004.

Feliciano, F. F. e Souza, I. L. de. Análise de Conformidade de Peças Através de Técnicas de Visão Computacional”. Projeto Final de Graduação. Curso de Engenharia Mecânica. Orientadora: Fabiana R. Leta UFF. Niterói. 2004.

Gomes, J. e Velho, L. Computação gráfica: Imagem. IMPA-SBM. 1994.

Khotanzad, A., Banerjee, H., Srinath, M. D. A., "Vision System for Inspection of Ball Bonds and 2-D Profile of Bonding Wires in Integrated Circuits”, IEEE Transactions on Semiconductor Manufacturing, Vol. 7, No. 4, pp. 413-422, 1994.

Leta, F. R., Feliciano, F. F. e Souza, I. L. de. Discussing accuracy in an automatic measurement system using computer vision techniques. 18th International Congress of Mechanical Engineering

ABCM, Novembro, Ouro Preto, MG, 2005.

Liguori, C., Paolillo, A., Pietrosanto, “An Automatic Measurement System for the Evaluation of Carotid Intima-Media Thickness”, IEEE Transactions on Instrumentation and Measurement, Vol. 50, No. 6, pp. 1684-1691, 2001.

Luo, P. F., Pan, S. P., Chu, T. C., "Application of computer vision and laser interferometer to the inspection of line scale”, Optics and Lasers in Engineering, Vol. 42, pp. 563-584, 2004.

Marques Filho, O. e Vieira Neto,H.. "Processamento Digital de Imagens," Ed. Brasport, 1999.

Ramesh, J.; Kasturi, R. e Schunk, B.. Machine Vision. New York, Ed. McGraw-Hill, 1995. 